\title{
El recibo electrónico de salario del empleado público
}

\author{
Antonio D. Berning Prieto \\ Universidad Pablo de Olavide, de Sevilla
}

SUMARIO: I.- PLANTEAMIENTO GENERAL. II.- EL REGIBO ELEGTRÓNICO DE SALARIO. III.- EL TRATAMIENTO DEL RECIBO ELECTRÓNICO DE SALARIO EN DEREGHO ADMINISTRATIVO Y SU IMPLANTACIÓN EN LAS ADMINISTRACIONES PÚBLICAS. IV.- LA INCORPORACIÓN DE LA FIRMA O CERTIFICADO ELECTRÓNICOS. V.- CONGLUSIONES. BIBLIOGRAFÍA

\section{RESUMEN}

El presente artículo pretende efectuar un acercamiento a la tradicional figura del recibo de salario y su aplicación en las relaciones empleador-trabajador en el seno de las Administraciones Públicas o entidades del sector público, así como al tratamiento que hasta la fecha se le ha conferido por el ordenamiento jurídico, la negociación colectiva y los Tribunales de Justicia cuando se ha pretendido su emisión en formato electrónico como forma de incrementar la eficacia en la gestión administrativa de la carga derivada de la gestión documental de la relación del propio personal a su servicio, examinándose los requisitos necesarios para una conversión al formato electrónico con garantías suficientes, para así conseguir una pacífica transición al nuevo formato digital del recibo de salario.

\section{ABSTRACT}

This article attempts to make an approach to the traditional figure of the wage bill and its application in employer-worker within the government or public sector entities relations and treatment to date has been conferred by the law, collective bargaining and the Courts of Justice when they have tried to issue electronic format as a way to increase efficiency in the administration of the burden of document management relationship for staff in service, examining the requirements for the conversion to electronic format with sufficient guarantees to achieve a peaceful transition to the new digital format of the salary receipt.

PALABRAS CLAVE

Empleado público, firma electrónica, recibo, salario, eficiencia. 


\section{KEYWORDS}

Civil service, electronic signature, receipt, salary, efficiency.

\section{PLANTEAMIENTO GENERAL}

La nueva era de la información ${ }^{1}$ supone el auge y, con ello, la implantación progresiva de las nuevas tecnologías en todas las esferas sociales, entre las que se encuentran los ciudadanos, las empresas y las propias Administraciones Públicas. Resulta incuestionable el enorme impacto que las nuevas tecnologías ha supuesto para todos los sectores de la sociedad, desde el usuario de la ya común telefonía móvil hasta el técnico especialista de la Administración Pública que interviene en la gestión de una licitación pública utilizando las plataformas electrónicas, consiguiendo una eficiencia y eficacia en la gestión de expedientes administrativos antes impensable.

En este nuevo estado de las cosas, resulta necesario adaptar el comportamiento social a esta nueva realidad, lo cual supone un proceso de adaptación física y psicológica, que no siempre es pacífico.

En el seno de las Administraciones Públicas es evidente el gran avance que ha supuesto la inversión y esfuerzos realizados en aras a la implantación de la Administración Electrónica, y los beneficios que ha traído consigo se hacen patentes cada día: reducción de gastos y cargas administrativas, evitación de largas colas y esperas, elevados beneficios medioambientales gracias a la reducción del gasto de papel, y así podría continuarse con un largo etcétera.

En definitiva, se consigue eficacia y eficiencia en la prestación de servicios públicos a los ciudadanos dándose cumplimiento así en mayor medida al mandato constitucional contenido en el artículo 103.1 CE, e incluso logros sociales y medioambientales. Pero la implantación de las nuevas tecnologías no sólo debe desplegar efectos ad extra, en las relaciones de la Administración con los ciudadanos ${ }^{2}$, sino que también resulta interesante que aquélla pueda agilizar y mejorar sus propios servicios ad intra ${ }^{3}$ gracias a la nueva realidad tecnológica, como es el caso de la implantación del recibo electrónico de salario.

\footnotetext{
1 Término acuñado por CASTELLS, M. en su obra "La era de la información. La sociedad red". Alianza Editorial, Madrid (2005).

${ }^{2}$ Conocido como G2C por las siglas en inglés de Government to Citizens.

${ }^{3}$ Denominándose en este caso G2E por las siglas de Government to Employees.
} 
Al tiempo que los avances tecnológicos suponen, en la mayor parte de los casos, una mejora en la prestación de los servicios públicos, al otorgarles notas de inmediatez y seguridad, a veces, no obstante, obligan a los sujetos intervinientes (funcionarios responsables de la tramitación de los procedimientos administrativos, destinatarios de los actos, etc.) a llevar a cabo un proceso de adaptación que no siempre es bienvenido, pues a veces encuentra obstáculos en el propio personal al servicio de las Administraciones Públicas al tener éstos por inalterables, en determinados supuestos, procedimientos que deben ser objeto de constante revisión en aras a la necesaria reducción de cargas al administrado y ahorro de costes a la Administración a través de la adopción de medidas de simplificación administrativa ${ }^{4}$, entre otras.

Es el caso de los recibos de salario, cuyo paso del formato papel al electrónico ha supuesto un evidente avance y ha tenido algunos sectores de oposición, lo cual ha llevado algunas causas ante los órganos jurisdiccionales competentes y ha dado lugar a determinados pronunciamientos judiciales, como se tendrá ocasión de examinar en el presente Trabajo.

El acierto en el fomento y consecución de estas medidas es evidente, si bien ello debe ir acompañado de medios suficientes que garanticen una correcta implantación de las mismas en aras a la salvaguarda, en este caso, de los derechos de los empleados públicos.

Por tanto, es objeto del presente Trabajo realizar un estudio del recibo electrónico de salario y la incorporación de un certificado electrónico al mismo como una de las manifestaciones del uso de las nuevas tecnologías en las relaciones laborales o funcionariales en el seno de las Administraciones Públicas y su influencia, por tanto, en el moderno Derecho del Trabajo y su necesaria relación con el Derecho Administrativo.

\section{EL REGIBO ELEGTRÓNICO DE SALARIO}

Dado que la liquidación y el pago del salario deben estar, como se verá, documentado, la forma tradicional de llevarlo a efecto es entregar al trabajador un reci-

\footnotetext{
${ }^{4}$ Para un estudio en mayor profundidad de la simplificación administrativa consúltese la obra coordinada por GAMERO CASADO, E. titulada "Simplificación del procedimiento administrativo y mejora de la regulación. Una metodología para la eficacia y el derecho a la buena administración”, Ed. Tirant Lo Blanch (Valencia, 2014).
} 
bo individual que justifique dicho abono, denominado recibo de salario. El hecho de que sea emitido en formato papel o electrónico supone simplemente trasladar el primero al ámbito de las nuevas tecnologías, manteniéndose indemne en todo caso la obligación del empresario, a la que tradicionalmente venía dando cumplimiento entregándolo en formato papel.

Con carácter general, debe traerse en primer lugar a colación el artículo 29 del Real Decreto Legislativo 2/2015, de 23 de octubre, por el que se aprueba el texto refundido de la Ley del Estatuto de los Trabajadores (en adelante, ET) el cual, en relación con la documentación de la liquidación y el pago de los salarios, únicamente dispone que se harán puntual y documentalmente en la fecha y lugar convenidos o conforme a los usos y costumbres, documentándose mediante la entrega al trabajador de un recibo individual y justificativo del pago del mismo que se ajustará al modelo que apruebe el Ministerio de Trabajo y Seguridad Social, salvo que por convenio colectivo o, en su defecto, por acuerdo entre la empresa y los representantes de los trabajadores, se establezca otro modelo que contenga una clara separación de las diferentes percepciones del trabajador, así como de las deducciones que legalmente procedan.

Como puede apreciarse, si bien se establecen in fine determinados requisitos de mínimos que la negociación colectiva debe respetar en todo caso, la entrega y contenido del recibo de salario se rige en primer lugar por lo convenido por mor de la negociación colectiva, ya se trate del convenio colectivo que resulta de aplicación, ya lo sea en virtud de acuerdo entre la empresa y los representantes de los trabajadores y, sólo en caso de ausencia de acuerdo, haciendo uso del modelo recogido normativamente.

El modelo de recibo de salario a que se hace referencia se encuentra en el Anexo de la Orden Ministerial de 27 de diciembre de 1994, por la que se aprueba el modelo de recibo individual de salarios, el cual ha sido modificado mediante la Orden ESS/2098/2014, de 6 de noviembre, con el fin de dar cumplimiento específicamente a lo previsto en el segundo párrafo del artículo 104.2 del texto refundido de la Ley General de la Seguridad Social, aprobado por el Real Decreto Legislativo 1/1994, de 20 de junio (actualmente debe entenderse referido al artículo 142 del recientemente publicado Real Decreto Legislativo 8/2015, de 30 de octubre, por el que se aprueba el texto refundido de la Ley General de la Seguridad Social), que fue añadido, con efectos de 1 de enero de 2013, por la disposición adicional vigésima sexta de la Ley 27/2011, de 1 de agosto, sobre actualización, adecuación y modernización del sistema de la Seguridad Social ya que, hasta su entrada en vigor, en los recibos de salarios de los trabajadores únicamente constaban la base de cotización y el tipo de retención correspondientes a la aportación del trabajador, pero no la determinación de la aportación del empresario. 
La precitada Orden de 1994 exige que el modelo de recibo de salario contenga, salvo que la negociación colectiva disponga algo distinto, la siguiente información:

- Identificación, tanto de la empresa como del trabajador.

- Periodo de liquidación referido a meses naturales, siendo cualquier liquidación inferior considerada como pago a cuenta.

- Desglose de todos los conceptos (abonos y retenciones), incluyendo indemnizaciones y suplidos.

- Indicación de la cuantía total de la cotización a la Seguridad Social, indicando la parte que corresponde a la aportación del empresario y la parte correspondiente al trabajador.

- Anticipos abonados previamente a cuenta de la liquidación definitiva.

- Firmas de la empresa y el trabajador.

Ninguna referencia se hace en la legislación vigente, por tanto, a la posibilidad de emitir el recibo de salario en formato electrónico ${ }^{5}$, constando únicamente que se hará "documentalmente" y que dicha documentación se hará "mediante la entrega al trabajador de un recibo individual y justificativo del pago", según modelo aprobado por la Orden anteriormente citada o el acordado en convenio colectivo o con los representantes de los trabajadores.

Se prevé que el empresario abone el importe del salario en metálico o por transferencia bancaria ${ }^{6}$, exigiendo en el primer caso la firma del trabajador en la nómina como medio justificativo de su abono, y eximiendo esta exigencia para el segundo caso, toda vez que se puede acreditar fácilmente por parte del empresario la realización de la misma, y sin que quepa en este caso la negativa del trabajador, puesto que la apertura de cuenta bancaria es una práctica aceptada generalmente en la sociedad actual $^{7}$.

${ }^{5}$ Lógicamente, en la fecha de aprobación de la Orden (1994) no se preveía el enorme avance que las tecnologías de la información y la comunicación proporcionarían. No obstante, tampoco con carácter posterior se ha incluido la posibilidad, lo cual hubiese sido a todas luces lo más deseable, ni tan siquiera en las recientes modificaciones normativas antes citadas.

${ }^{6}$ La única limitación al respecto la establece el artículo 29.4 del ET, al exigir la comunicación a los representantes de los trabajadores del medio escogido por el empresario.

${ }^{7}$ Afirmación puesta de manifiesto por NIETO ROJAS, P. en "El recibo de salario en formato electrónico", Revista de la Contratación Electrónica n ${ }^{\circ} 114$ (2011), p. 87. 
La casuística ha puesto de manifiesto determinados aspectos relacionados con los requisitos anteriormente citados del recibo de salario. Por ejemplo, aun firmándose por parte del trabajador el recibo de salario, ello únicamente hace prueba del abono de lo consignado en el mismo ${ }^{8}$, pudiendo por tanto reclamar posteriormente conforme estime oportuno, y sin que la falta de sello de la empresa les haga perder su condición de auténticos ${ }^{9}$.

Ahora bien, desde hace unos años han venido sucediéndose algunas sentencias dictadas en relación con la validez del recibo de salario en formato electrónico, que se caracterizan por ser, usualmente, contrarias a su establecimiento en lugar del formato papel.

No obstante, algún pronunciamiento jurisprudencial como el contenido en la sentencia 8076/2006, de 20 de noviembre, del Tribunal Superior de Justicia de Cataluña ${ }^{10}$, contiene un razonamiento totalmente flexible respecto a la documentación electrónica del recibo de salarios, y confirma el pronunciamiento judicial de instancia que se había recurrido por los trabajadores, al afirmar que "[...] desde el año 2003 la empresa demandada ha implantado a nivel nacional una aplicación informática denominada e-domus, en virtud de la cual cada trabajador puede acceder a su nómina e imprimirla desde su ordenador personal a través de una clave individualizada de acceso. La nómina que se consulta on-line no se recibe en soporte papel; y aun teniendo ambas idéntico contenido si la parte actora solicita un certificado de salarios a la empresa ésta lo expide [...]", por lo que concluye que "no puede considerarse vulnerada la norma cuya infracción se denuncia en tanto que <la empresa efectúa el pago de las nóminas de los actores mediante transferencia bancaria $>$ (esto es, en los términos que aquélla permite); sin que se encuentre <legalmente $>$ obligada a documentar por escrito unos $\langle$ recibos $>$ de salarios sustituidos (en su garantista finalidad) por el -confidencial-conocimiento on-line $<d e$ los diferentes conceptos de abono>".

Este pronunciamiento ha merecido el elogio de cierto sector doctrinal ${ }^{11}$, al ser totalmente compatible con la realidad tecnológica actual y acoger la finalidad y espíritu de las normas, bajo el acertado prisma de que el recibo de salario en soporte informático y papel es idéntico.

\footnotetext{
${ }^{8}$ Ex artículo 3.5 del ET.

9 Vid. sentencia 232/1997, de 14 de mayo, del Tribunal Superior de Justicia de Navarra (AS $1997 \backslash 1624)$.

${ }^{10}$ AS 2007\} \backslash 1 0 6 0 \text { (Rec. n 331/2005) }

${ }^{11}$ LLOMPERT BENNÀSSAR, M. "El recibo de salario en formato electrónico". Revista Doctrinal Aranzadi Social n 2 (2007), p. 2765 (BIB 2007\1352).
} 
Posteriormente, la sentencia 121/2010, de 9 de diciembre, de la Audiencia Nacional $^{12}$ negó la validez del cambio adoptado por la empresa de poner a disposición en la intranet (red local) el recibo de salario en lugar de entregarlo en formato papel por diversos motivos (negación reiterada en la posterior sentencia del mismo órgano jurisdiccional 76/2015, de 28 de abril ${ }^{13}$ ), destacando determinados pronunciamientos relativos a que (i) la entrega del recibo de salario es una obligación de hacer que, conforme al artículo 1166 del Código Civil (CG, en adelante), no puede entenderse cumplida con su mera puesta a disposición a los trabajadores; (ii) no existe acuerdo individual ni colectivo al respecto; (iii) la negativa del trabajador a recibirla en formato electrónico no es contraria a la buena fe, pues según el órgano jurisdiccional no tiene la misma facilidad de uso que el formato en papel; (iv) la documentación del recibo de salario en formato electrónico sin sello ni firma de la empresa no tiene los mismos efectos probatorios que en papel, gozando éste de ventajas respecto a aquél ${ }^{14}$.

El anterior pronunciamiento fue confirmado por el Tribunal Supremo mediante sentencia de 22 de diciembre de $2011^{15}$ con motivo del recurso de casación planteado por la empresa, llegando incluso a afirmar el Tribunal que "[...] el instrumento documental previsto en la legislación, que es sin duda, a la vista de los preceptos reproducidos, el recibo en soporte papel. Al recibo en soporte papel se refiere el artículo 29.1 párrafo $3^{\circ}$ ET (RCL 1995, 997) cuando habla de <recibo individual>, e incluso con más claridad a tal documento en papel se refiere también la Orden Ministerial cuando precisa que lo que se entrega al trabajador es el $<$ duplicado > del recibo".

Los pronunciamientos de ambos órganos jurisdiccionales no merecen, por supuesto, elogio alguno, al encontrarse totalmente desfasados y descontextualizados a pesar de la fecha en que han sido dictados, siendo totalmente cuestionables, por cuanto: (i) en relación con el hecho de que se trata de una obligación de hacer por parte del empresario, nada obsta a que éste de cumplimiento a la misma poniendo a disposición del trabajador el documento para su descarga en formato electrónico, más aún cuando además de dicha obligación principal también cumple con la accesoria de poner medios a disposición de aquéllos para ello; (ii) la inexistencia de acuerdo individual o colectivo no es óbice para que los recibos de salario se emitan en for-

\footnotetext{
${ }^{12}$ JUR $2010 \backslash 416611$ (Rec. no 178/2010)

${ }^{13}$ JUR 2015 \134002 (Pcdto. no 36/2015)

${ }^{14}$ En los mismos términos se pronuncia la anterior sentencia 41/2005, de 27 de abril, de la Audiencia Nacional (JUR 2005\232032, Rec. $n^{\circ}$ 8/2005).

${ }^{15}$ RJ $2012 \backslash 1887$ (Rec. no 3/2011)
} 
mato electrónico, toda vez que se trata simplemente de la forma de cumplimiento de la obligación y la sociedad actual se encuentra perfectamente habilitada para hacer uso de tales formatos de documentos tras su descarga, e incluso la sentencia de 5 de abril de 1993, del Tribunal Superior de Justicia de Murcia ${ }^{16}$ concluyó, en relación con la necesidad de llegar a acuerdo con los representantes de los trabajadores para modificar el recibo de salario, que tratándose de cambios o modificaciones necesarias para adaptar el recibo de salario a la normativa legal o al convenio colectivo de aplicación al tratarse de una facultad empresarial inherente al poder de dirección del empresario, la cual ha llevado a cierta doctrina ${ }^{17}$ a efectuar, con acierto, una interpretación analógica de dicho pronunciamiento judicial en relación con la modificación del formato de entrega del recibo de salario al electrónico, en el sentido de que no es necesario efectuar consulta con los representantes de los trabajadores, siempre y cuando se informe convenientemente de la medida adoptada empresarialmente; (iii) que la negativa del trabajador no sea contraria a la buena fe por la mayor facilidad del formato papel dependerá del ámbito sectorial en que nos encontremos, pues un trabajador del sector de las telecomunicaciones tendrá incluso más facilidad para recibir y almacenar el documento en formato electrónico que si se tratase del formato papel; y (iv) se da cumplimiento al principio de equivalencia de soportes con la incorporación de la firma o sello electrónico al recibo de salario, como se verá.

Como puede apreciarse, estos últimos pronunciamientos comentados, a pesar de tener un (cuestionable) sustento teórico-jurídico, no se ajustan en absoluto al estado actual de la técnica y la tecnología, ya que los avances existentes y la incorporación de ésta a las relaciones de trabajo permiten la sustitución de abundante documentación, que antes únicamente se documentaba en papel, por el formato electrónico, como se ha llevado a la práctica también exitosamente en materia de Seguridad Social ${ }^{18}$. Incluso algún autor ${ }^{19}$ ha puesto en entredicho, acertadamente, al co-

${ }^{16}$ AS $1993 \backslash 2119$ (Rec. no 103/1993)

17 GÓMEZ-MILLÁN HERENCIA, M.J. "El recibo de salario en formato electrónico. Comentario de la sentencia del Tribunal Supremo de 22 de junio de 2011”. Boletín de Función Pública del Instituto Nacional de Administración Pública n 7 (2012), p. 50.

${ }^{18}$ Piénsese cómo todas las comunicaciones efectuadas entre las empresas y la Administración en materia de Seguridad Social se efectúan por vía electrónica desde hace años (vid. al respecto la Resolución de la Dirección General de la Seguridad Social, de 23 de mayo de 1995, que desarrolla la Orden de 3 de abril anterior, sobre uso de medios electrónicos, informáticos y telemáticos en relación con la inscripción de empresas, afiliación, altas y bajas de trabajadores, cotización y recaudación en el ámbito de la Seguridad Social, en especial su Anexo V), habiendo supuesto un evidente avance y ahorro de tiempo y dinero en la gestión documental para ambas partes, todo ello sin mencionar, por supuesto, los evidentes benefi- 
mentar la sentencia precitada, que la solución adoptada fuese la definitiva, ya que al ser posible actualmente la sustitución del formato papel por el electrónico con todas las garantías exigibles será necesario que "empresas, representantes de los trabajadores y poderes normativos hagan sus deberes" ${ }^{\prime 20}$.

Desde luego, la interpretación llevada a cabo por la Audiencia Nacional en su pronunciamiento no se corresponde en absoluto con la realidad imperante actualmente. Nótese cómo una sentencia dictada años antes, concretamente el 14 de mayo de $1997^{21}$, por el Tribunal Superior de Justicia de Navarra, seguía un criterio mucho más correcto, al mantener que la ausencia de firma y sello de la empresa no le priva al recibo de salario (referido al formato papel) de su condición de auténtico para ser valorado, lo cual no viene sino a confirmar que se torna necesaria una adaptación de la regulación normativa existente, que se encuentra obsoleta completamente en este aspecto, así como por empresarios, trabajadores, representantes de éstos y el propio Estado legislador.

cios que ello trae consigo para materias tan sensibles como el medio ambiente y la sostenibilidad, por lo que además de suponer un beneficio para los entes privados, el interés público se erige en beneficiario directo de tales medidas. Nótese cómo lo pone de manifiesto NIETO ROJAS, P. (2011): 85, al apuntar la progresiva utilización de herramientas telemáticas por parte de empresas gracias a su impulso por la Administración Pública, concretamente por tratarse del sector de referencia la Tesorería General de la Seguridad Social, con el sistema RED para las comunicaciones de altas y bajas, el DELTA para el tratamiento de accidentes de trabajo, y el Contrat@, para las comunicaciones en materia de contratos de trabajo, por citar algunos. Debe tenerse en cuenta, además, que el actual avance de la tecnología ha permitido que desde el pasado 1 de enero la Tesorería General de la Seguridad Social (TGSS) haya puesto en funcionamiento el nuevo Sistema de Liquidación Directa de Cotizaciones a la Seguridad Social (conocido como "Proyecto Cret@"), asumiendo un papel activo en el proceso de recaudación a través de la modificación del modelo de autoliquidación al de facturación, de manera que será la TGSS la que envíe un borrador de liquidación a las empresas, que podrán no obstante efectuar modificaciones o correcciones y remitirlas de nuevo a la Administración, que tiene como objetivos prioritarios minimizar los errores mediante la aplicación de las reglas de cotización por parte de la TGSS, contraste de los datos con carácter previo a la liquidación y eliminando la necesidad de actuaciones presenciales, dando clara preponderancia a la vía electrónica, telemática o informática.

${ }^{19}$ SEMPERE NAVARRO, A.V.: "Entrega del recibo de salarios en papel”. Revista Aranzadi Doctrinal, $n^{\circ} 11$ (2012). Ed. Thomson Reuters Aranzadi, p. 15 (BIB 2012\978).

20 Tales "deberes" no son más que hacer un esfuerzo por adaptar su actuación y fomentar en la medida de lo posible este tipo de medidas positivas. Comparto absolutamente que existe una evidente necesidad, que debe ser abordada por el poder normativo, de efectuar una adaptación de la regulación del recibo de salario, ya que la vigente data de hace más de veinte años, para adaptarlo a la realidad existente actualmente.

\author{
Revista Andaluza de Administración Pública
}

ISSN: 1130-376X, núm. 93, Sevilla, septiembre-diciembre (2015), págs. 367-388 


\section{EL TRATAMIENTO DEL RECIBO ELEGTRÓNICO DE SA- LARIO EN DEREGHO ADMINISTRATIVO Y SU IMPLAN- TACIÓN EN LAS ADMINISTRACIONES PÚBLICAS}

Procede examinar a continuación si en el seno de las Administraciones Públicas, en las que se impulsa desde hace años la implantación de una efectiva Administración Electrónica, es posible sustituir el recibo de salario en formato papel por el electrónico para el personal que le presta sus servicios, sobre la base de la situación actual de la cuestión que se ha tenido ocasión de examinar.

En primer lugar, debe advertirse la existencia en el seno de las Administraciones Públicas de personal a su servicio cuya vinculación con la misma puede variar, al coexistir funcionarios de carrera, interinos, personal laboral (fijo, por tiempo indefinido o temporal) y eventual ${ }^{22}$, a los que debe adicionarse el personal directivo regulado en el artículo 13 del Real Decreto Legislativo 5/2015, de 30 de octubre, por el que se aprueba el texto refundido de la Ley del Estatuto Básico del Empleado Público (en adelante, también TREBEP).

$\mathrm{Al}$ igual que en el elenco normativo que conforma el Derecho del Trabajo no existe referencia alguna, tampoco se ha regulado el recibo electrónico de salario por el de la Función Pública. Curiosamente, la única referencia legislativa a la documentación electrónica del recibo de salario $^{23}$ en el ámbito jurídico-público se encuentra recogida en el artículo 7 del Real Decreto 598/1985, de 30 de abril, sobre incompatibilidades del personal al servicio de la Administración del Estado, de la Seguridad Social y de los Entes, Organismos y Empresas dependientes, al establecer la obligación a los Directores Generales de quienes dependían las habilitaciones de personal que satisficieren cualquier tipo de retribución periódica con cargo a los presupuestos de la Administración del Estado y de la Seguridad Social, Organismos autónomos y Entes o Empresas dependientes, a remitir anualmente a la Inspección General de Servicios de la Administración Pública el soporte magnético o la copia de la

21 AS 1997\1624

22 Considerándoseles a todos ellos "empleados públicos" ex artículo 8 del Real Decreto Legislativo 5/2015, de 30 de octubre, por el que se aprueba el texto refundido de la Ley del Estatuto Básico del Empleado Público.

${ }^{23}$ Debe advertirse que únicamente es una referencia a su almacenamiento, ya que no se prevé la entrega al trabajador del mismo, sino únicamente a efectos de su conservación. Parece estar refiriéndose a aquellos casos en que se ha procedido por parte de la Administración a la digitalización de la nómina entregada previamente en formato papel, sobre todo teniendo en cuenta que el citado Reglamento data de 1985, anterior incluso al propio ET o a la Orden de 1994 que regula el contenido del recibo de salario. 
nómina de las retribuciones mensuales que establecía la Secretaría de Estado para la Administración Pública.

Puede afirmarse, por tanto, que existe un precedente legislativo en el que, al menos, se tiene en cuenta la posibilidad de que la nómina se encuentre almacenada en formato electrónico ${ }^{24}$, aunque no de la forma que permite el estado de la técnica ni con la operatividad y seguridad que actualmente ha llegado a alcanzar.

No obstante, el actualmente vigente Capítulo III del TREBEP, relativo a los derechos retributivos, recoge en sus artículos 21 a 30 los conceptos salariales y su regulación, tales como las retribuciones básicas, complementarias, indemnizaciones, etc., pero sin precisión alguna en relación con la documentación de tales percepciones salariales por lo que no se regula, ni siquiera para los funcionarios de carrera, un modo distinto al previsto en la Orden de 1994.

En relación a su implantación debe tenerse en cuenta en primer lugar al respecto lo previsto en el Capítulo IV del TREBEP, relativo al derecho a la negociación colectiva, representación y participación institucional, puesto que la potestad normativa del empresario público no puede sustituir a la negociación colectiva, ya que en tal caso serían impugnadas las resoluciones y disposiciones reglamentarias dictadas sin previo intento de negociación e irremediablemente anuladas, como se puede comprobar en la sentencia de 27 de octubre de 2014 del Tribunal Supremo ${ }^{25}$. Lo más conveniente es el establecimiento previo de Mesas de Negociación en las que se pacte o acuerde colectivamente el contenido de las normas que posteriormente se aprueben para regular las relaciones funcionariales o laborales del personal al servicio de la Administración.

Aunque algún pronunciamiento jurisprudencial posterior del Tribunal Supremo en relación con la implantación del recibo de salario en el ámbito de las Administraciones Públicas ha confirmado el previo pronunciamiento de algún Tribunal Superior de Justicia proclive a la sustitución, lo cierto es que ha sido fundamentado en la falta de determinados requisitos procesales propios del recurso de casación, no por motivos de fondo de los que colegir la existencia de un cambio de criterio jurisprudencial, que deviene inexistente en este aspecto.

\footnotetext{
${ }^{24}$ Téngase en cuenta que se hace referencia al "soporte magnético", lo cual responde a la realidad imperante en aquél momento, en que los dispositivos de almacenamiento extraíbles (y, por ende, trasladables) eran, fundamentalmente, disquetes de 5¹/4 y 31/2 (cuya invención correspondió a IBM en 1976 y 1983 , respectivamente).

25 RJ 2014\5413, (Rec. n 3452/2013)
} 
Así sucedió concretamente en el supuesto ventilado en la sentencia 5075/2011, de 22 de junio, del Tribunal Supremo ${ }^{26}$, que resuelve un recurso de casación promovido por la Central Sindical Independiente y de Funcionarios contra la anterior sentencia de 8 de octubre de 2010, del Tribunal Superior de Justicia de Asturias, que desestimó a su vez la demanda de conflicto colectivo planteada por aquél contra la decisión de la Consejería de Administraciones Públicas y Portavoz del Gobierno del Principado de Asturias consistente en, precisamente, implantar un sistema electrónico de recibo de salario que sustituye al papel.

En dicho supuesto la decisión del empresario público estaba revestida de todo tipo de garantías y previsiones, abarcando reuniones informativas previas (tanto con el personal como con los representantes de los trabajadores), entrega de información detallada con la última nómina en formato papel, establecimiento de un sistema de acceso a la intranet mediante claves personales (incluso para aquellos trabajadores que no se encontraban ya en activo), posibilidad de acceso de cada trabajador desde su ordenador personal y, para aquellos que experimentasen problemas o no tuviesen un ordenador personal para acceder ${ }^{27}$, se mantenía la posibilidad de imprimirlo y entregárselo en papel previa solicitud.

Lógicamente, el Alto Tribunal entendió que existe una plena equivalencia de soportes, sin que la adopción de una u otra forma sea óbice para el cumplimiento de su obligación por parte del empresario (público o privado) y sin que la mera reticencia de trabajadores o sindicatos a la adopción de este nuevo formato por simples formalidades o interpretaciones literales de la normativa vigente puedan dar lugar a una directa inadmisión del recibo de salario en formato electrónico por el simple hecho de no entregarse en papel.

${ }^{26}$ RJ $2011 \backslash 5945$ (Rec. n ${ }^{\circ}$ 193/2010)

${ }^{27}$ Los problemas que podría conllevar el acceso a este tipo de información a través de ordenadores de uso colectivo han sido puestos de manifiesto por GÓMEZ-MILLÁN HERENCIA, M.J. (2012): 54, con la acertada advertencia de que no sería igual de garantista si el acceso a la nómina se permite de tal forma, si bien dicha modalidad ha sido aceptado por el Tribunal Superior de Justicia de Murcia en su sentencia 288/2008, de 26 de mayo (JUR 2008\322016, Rec. $n^{\circ}$ 208/2008), a pesar de que, a juicio de la citada autora, ello podría dar lugar a vulneraciones de los datos de carácter personal del trabajador por cuanto determinados archivos temporales son alojados en el dispositivo y algún usuario posterior con conocimientos informáticos podría acceder a ellos. Tal obstáculo podría salvarse con la simple instalación de un software adecuado que elimine todo archivo temporal una vez cerrada la sesión del usuario trabajador, pudiendo automatizarse o llevarse a cabo de forma manual por el trabajador una vez concluya la descarga, debiéndose en tal caso incluir en las instrucciones que se proporcionen. 
El recibo de salario, con carácter general en el ámbito de las Administraciones Públicas, ostenta la naturaleza jurídica de acto administrativo, concretamente podría considerarse, si se observa la clasificación de los actos en función del número de destinatarios $^{28}$, como singular, por tener un único destinatario, aunque se dicte para ser notificado a todos los trabajadores que prestan servicios a la Administración de que se trate, puesto que tal notificación es personal y distinta del resto, pues la retribución también lo es, configurándolo la propia jurisprudencia, en definitiva, como un acto administrativo de contenido informativo ${ }^{29}$.

Por su parte, en el ámbito concreto de las Administraciones Públicas, el TREBEP no establece ninguna previsión expresa respecto a la forma de entrega del recibo de salario, por lo que habrá de efectuarse un examen sistemático de la regulación de la cuestión en la normativa que resulta de aplicación y el ET como norma de aplicación directa al personal laboral. En relación con la normativa aplicable, debe tenerse en cuenta que el artículo 2 del TREBEP dispone que el mismo "se aplica al personal funcionario y en lo que proceda al personal laboral', mientras que el ET establece en su artículo 1.3.a) la exclusión de su ámbito de aplicación de la relación de servicio de los funcionarios públicos, que se regulará por el TREBEP cuando dicha relación se regule por normas administrativas o estatutarias.

Ahora bien, toda vez que el recibo de salario no se encuentra regulado en el TREBEP, supletoriamente debe aplicarse lo dispuesto en el ET, puesto que de lo contrario supondría dejar vacía de contenido la obligación del empresario (público en este caso) y en situación de desigualdad a los funcionarios respecto al personal laboral ex artículo $14 \mathrm{CE}$, pues éste contaría con su propia regulación (relativamente exigente además) respecto al contenido del recibo de salario y no así los funcionarios públicos ${ }^{30}$, salvo previsión expresa al respecto derivada de la negociación colectiva.

${ }^{28}$ Clasificación a la que hacen referencia GAMERO CASADO, E. y FERNÁNDEZ RAMOS, S. en su excelente y pedagógica obra rubricada "Manual básico de Derecho Administrativo", Ed. Tecnos, $11^{\text {a }}$ ed. (2014), pp. 434-435.

${ }^{29} \mathrm{Vid}$. Fundamento Jurídico Tercero de la sentencia 5075/2011, de 22 de junio, del Tribunal Supremo, citada anteriormente (RJ 2011\5945, Rec. nº 193/2010).

${ }^{30}$ En relación con la aplicación práctica del TREBEP y el ET puede consultarse el documento elaborado por la Secretaría General para la Administración Pública del Ministerio de Administraciones Públicas rubricado "Instrucciones para la aplicación del Estatuto Básico del Empleado Público en el ámbito de la Administración General del Estado y sus Organismos Públicos", aprobado mediante Resolución de 21 de junio de 2007, publicado en el Boletín Oficial del Estado n ${ }^{\circ}$ 150, de 23 de junio de 2007, pp. 27.178 a 27.183. 
En primer lugar, en relación con la equivalencia de los formatos papel y electrónico, debe tenerse en cuenta lo previsto en la Ley 11/2007, de 22 de junio, de Acceso Electrónico de los Ciudadanos a los Servicios Públicos (LAE, en adelante), en especial los principios generales que resultan de aplicación ex lege al ámbito de la Administración Electrónica, sin que aparezca expresamente reconocido el esencial principio de equivalencia funcional entre el documento emitido en papel y el electrónico.

No obstante, como ha tenido ocasión de afirmar la doctrina ${ }^{31}$, dicho principio se puede inteligir de una serie de reglas, en especial del artículo 45.5 de la LRJPAC, que reconoce la plena validez y eficacia de los documentos emitidos por medios electrónicos, informáticos o telemáticos por las Administraciones Públicas en cualquier tipo de soporte, así como los que éstas emitan como copias de originales almacenados por estos mismos medios siempre que quede garantizada su autenticidad, integridad y conservación y, en su caso, la recepción por el interesado, así como el cumplimiento de las garantías y requisitos exigidos legalmente.

Por tanto, habrá de procederse en primer lugar a examinar si concurren los requisitos que la LRJPAC ${ }^{32}$, como norma de carácter básico, y la LAE establecen para determinar la validez del documento electrónico que incorpora el recibo de salario. Una vez entren en vigor las Leyes 39 y 40/2015, de 1 de octubre,de Procedimiento Administrativo Común de las Administraciones Públicas (LPAC) y del Régimen Jurídico del Sector Público (LRJSP), respectivamente, habrán de tenerse en cuenta sus específicas previsiones, que serán mencionadas cuando proceda a lo largo del presente trabajo.

El documento electrónico es definido en el apartado j) del Anexo a la LAE, como aquella "información de cualquier naturaleza en forma electrónica, archivada en un soporte electrónico según un formato determinado y susceptible de identificación y tratamiento diferenciado", definición que se recoge también en el artículo 3.5 de la LFE, si bien el apartado 6 de dicho precepto concreta qué caracteres deben reunir para ser considerados documentos "públicos" o "administrativos".

${ }^{31}$ En este sentido, GAMERO CASADO, E. y VALERO TORRIJOS, J. (Coords.), en "La Ley de Administración Electrónica. Comentario sistemático a la Ley 11/2007, de 22 de junio, de Acceso Electrónico de los Ciudadanos a los Servicios Públicos", Ed. Thomson Reuters Aranzadi, 3a ed. (2010), pp. 153-156.

${ }^{32}$ Debe tenerse en cuenta que se encuentra en periodo de vacatio legis la nueva Ley 39/2015, de 1 de octubre, de Procedimiento Administrativo Común de las Administraciones Públicas (y la Ley 40/2015, de 1 de octubre, de Régimen Juídico del Sector Público, si bien esta regula aspectos mayormente organizativos o de relaciones ad intra de la Administración), que derogará la LRJPAC y la LAE en su intención de integrar en un mismo cuerpo legal ambas, si bien la exposición aquí contenida no se ve modificada por 
La LRJPAC (artículo 46 y concordantes) interpretada en relación con la LAE (artículo 29) supedita la validez y eficacia del documento electrónico, así como la total verificación del principio de equivalencia funcional ${ }^{33}$ respecto al documento original, a que (i) quede garantizada su autenticidad, integridad y conservación, (ii) la recepción por el interesado en los casos en que sea necesario y (iii) el cumplimiento de las garantías y requisitos exigidos por las Leyes.

Por su parte, el artículo 29.1 de la LAE establece la validez del documento administrativo electrónico "siempre que incorporen una o varias firmas electrónicas", lo cual es reiterado por el artículo 41.1 del Real Decreto 1671/2009, de 6 de noviembre, por el que se desarrolla parcialmente la Ley 11/2007, de 22 de junio, de Acceso Electrónico de los Ciudadanos a los Servicios Públicos (en adelante, RLAE) ${ }^{34}$, al establecer las características que debe reunir el documento electrónico que, como apunta la doctrina $^{35}$, constituyen requisitos para su validez además de: a) contener información de cualquier naturaleza; b) estar archivada la información en un soporte electrónico según un formato determinado y susceptible de identificación y tratamiento diferenciado; c) disponer de los datos de identificación que permitan su individualización, sin perjuicio de su posible incorporación a un expediente electrónico; y d) ser expedidos y firmados electrónicamente mediante los sistemas de firma previstos en los artículos 18 y 19 de la LAE. Debe tenerse en cuenta al respecto que el artículo 26.1 de la LPAC añade, como requisitos de validez de los actos administrativos en soporte electrónico, que incorporen una referencia temporal del momento en que han sido

ello, a excepción de los preceptos y norma de cobertura que resultarán de aplicación una vez se produzca su entrada en vigor el próximo mes de octubre de 2016.

${ }^{33}$ En el ámbito jurídico-privado, este principio ha sido reconocido ex lege por el artículo 23.3 de la Ley 34/2002, de 11 de julio, de Servicios de la Sociedad de la Información y del Comercio Electrónico, que dispone "siempre que la Ley exija que el contrato o cualquier información relacionada con el mismo conste por escrito, este requisito se entenderá satisfecho si el contrato o la información se contiene en un soporte electrónico". Si bien en el ámbito de la Administración Pública podría algún objetor invocar el apartado 4 in fine del mismo precepto comentado, que establece que "los contratos, negocios o actos jurídicos en los que la Ley determine para su validez o para la producción de determinados efectos la forma documental pública, o que requieran por Ley la intervención de órganos jurisdiccionales, notarios, registradores de la propiedad y mercantiles o autoridades públicas, se regirán por su legislación específica", el que suscribe entiende que no resulta de aplicación al recibo de salario, por no alcanzar éste la condición de "documento público"; pero es más, aun entendiéndolo como tal, operaría la remisión a la normativa específica, que como se examinará prevé la producción de actos administrativos en formato electrónico con plena validez como si del formato papel se tratase.

${ }^{34}$ Debe tenerse en cuenta que el mismo resulta de aplicación, en principio, a la Administración General del Estado.

35 SANZ LARRUGA, F.J., "Documentos y archivos electrónicos", en GAMERO CASADO, E. y VALERO TORRIJOS, J. (2010): 699. 
emitidos (apartado c) -nótese que no se refiere a un sello de tiempo electrónico emitido por una autoridad de sellado de tiempo (TSA), como hubiese sido deseable, sino a una mera referencia temporal- y los metadatos mínimos exigidos (apartado d).

El recibo de salario en formato electrónico, considerado como documento electrónico, reúne las características que exige dicha norma, al contener información de naturaleza salarial, encontrarse archivado en formato determinado (normalmente “.pdf”, aunque no es necesariamente el único que podría utilizarse) susceptible de identificación y tratamiento diferenciado, y es posible su individualización, al ser personal e individual su entrega, debiendo además incorporar, de acuerdo con el apartado 2 del precepto anteriormente transcrito, la correspondiente firma electrónica.

Procede por tanto examinar si los requisitos de autenticidad, integridad y conservación podrían cumplirse mediante la utilización de la firma o certificado electrónicos gracias al software informático adecuado, aspectos que se examinarán a continuación y de cuyo resultado dependerá si efectivamente resulta viable y suficientemente garantista sustituir el recibo de salario en papel por el electrónico en el seno de las Administraciones Públicas españolas.

\section{LA INCORPORACIÓN DE LA FIRMA O GERTIFICADO ELECTRÓNICOS}

Partiendo de la base de que el recibo electrónico de salario debe reunir determinados requisitos de carácter material (devengos, deducciones, anticipos o abonos a cuenta de la liquidación mensual correspondiente, etc.) y formal (firma y sello de la empresa -téngase en cuenta que se excepciona la firma del trabajador cuando se efectúa el abono de nómina por transferencia bancaria, método generalizado en la actualidad-), debe examinarse si el formato electrónico garantiza el cumplimiento de los requisitos de autenticidad, integridad y conservación, para preservar los derechos y garantías de los interesados (en este caso trabajadores adscritos a una determinada Administración Pública, lo que supone bien una relación de carácter laboral, bien una relación de especial sujeción como personal funcionario ${ }^{36}$ ) a través de sistemas electrónicos creados por la Administración Pública ad hoc, ya que deben ofrecer las mismas garantías e idéntica seguridad jurídica a sus destinatarios que el anterior sistema vigente (formato papel y entrega física del documento).

${ }^{36}$ Puede consultarse, respecto a la heterogeneidad en el empleo público, SÁNCHEZ MORÓN, M. en "Derecho de la Función Pública". Ed. Tecnos, 8a ed. (2014), pp. 65 y ss., en especial pp. 68 y ss. 
Por tanto, se torna necesario examinar si la firma o certificado electrónicos ${ }^{37}$ incorporados al documento electrónico que conforma la nueva versión del recibo de salario puede dar respuesta a tales exigencias.

Con carácter general, la firma electrónica puede definirse, de acuerdo con el artículo 3.1 de la Ley 59/2003, de 19 de diciembre, de Firma Electrónica (LFE, en adelante), como "el conjunto de datos en forma electrónica, consignados junto a otros o asociados con ellos, que pueden ser utilizados como medio de identificación del firmante", pudiendo llegar a tener la consideración de "avanzada" si "permite identificar al firmante y detectar cualquier cambio ulterior de los datos firmados, que está vinculada al firmante de manera única y a los datos a que se refiere y que ha sido creada por medios que el firmante puede mantener bajo su exclusivo control" y pudiendo ser la firma electrónica avanzada, a su vez, "reconocida", siempre y cuando esté "basada en un certificado reconocido y generada mediante un dispositivo seguro de creación de firma", caso en el que opera plenamente el principio de equivalencia funcional ope lege ex artículo 3.4 de la LFE, al disponer que "tendrá respecto de los datos consignados en forma electrónica el mismo valor que la firma manuscrita en relación con los consignados en papel'.

Pues bien, la firma electrónica utiliza un sistema criptográfico formado por dos claves, una pública y una privada, que gracias a la función hash permite firmar un documento, encriptarlo y garantizar a la persona que lo desencripte (destinatario) que:

Es auténtico (principio de autenticidad), es decir, que el órgano administrativo -en este caso- es el autor del documento, con lo cual también se permite comprobar su competencia.

${ }^{37}$ Debe tenerse en cuenta, en relación con la regulación de la firma electrónica, que en el seno de la Unión Europea se ha dictado el Reglamento (UE) 910/2014, del Parlamento Europeo y del Consejo, de 23 de julio de 2014, relativo a la Identificación Electrónica y los Servicios de Confianza para las Transacciones Electrónicas en el Mercado Interior y por la que se deroga la Directiva 1999/93/CE sobre firma electrónica, el cual parte de la premisa de que existe un clima de desconfianza en los particulares, empresas y Administraciones Públicas, por lo que éstos perciben cierta inseguridad jurídica que impide la adopción de la forma electrónica como medio seguro de comunicación y celebración de negocios jurídicos, por lo que la norma pretende establecer un clima de confianza en el entorno europeo para conseguir un pleno desarrollo económico y social en la Unión, si bien debe tenerse en cuenta que no resulta de aplicación al supuesto que nos ocupa ex artículo 2 del Reglamento, toda vez que, como apunta el Considerando (21) del mismo, éste "no debe cubrir la prestación de servicios utilizados exclusivamente dentro de sistemas cerrados entre un conjunto definido de participantes, que no tengan efectos en terceros. Por ejemplo, los sistemas establecidos en empresas o administraciones públicas para gestionar procedimientos internos que hagan uso de servicios de confianza". 
Su contenido es íntegro (principio de integridad), que demuestra que el mismo no ha sido alterado desde que fue creado por su autor ni se ha excluido ningún paquete de datos durante su envío o recepción ${ }^{38}$ (principio de inalterabilidad).

En cuanto a la forma de producirse los actos o recibos de salario, éstos se generarán de forma automatizada, ya que el software de gestión de nóminas de que disponga la Administración en cuestión se encargará de emitirlos "en masa", por lo que habrá de examinarse la regulación concreta prevista para este tipo de actos. En este sentido, el artículo 18 de la LAE (en similares términos se encuentra redactado el artículo 41 de la LRJSP, si bien en el caso del sello electrónico se precisa que deberá estar basado en un certificado electrónico o cualificado -denominación esta última proveniente del Reglamento (UE) 910/2014-) prevé que pueda llevarse a cabo la identificación y autenticación del ejercicio de la competencia en la citada actuación administrativa automatizada, mediante los siguientes sistemas de firma electrónica:

a) Sello electrónico de Administración Pública, órgano o entidad de derecho público, basado en certificado electrónico que reúna los requisitos exigidos por la legislación de firma electrónica.

b) Código Seguro de Verificación (CSV) vinculado a la Administración Pública, órgano o entidad y, en su caso, a la persona firmante del documento, permitiéndose la comprobación de la integridad del documento mediante el acceso a la sede electrónica correspondiente.

Por tanto, puede afirmarse que existen dos formas posibles que verifican los requisitos anteriormente reseñados para la válida emisión del recibo de salario en formato electrónico en el seno de las Administraciones Públicas, entre ellas el objeto de examen, esto es, el sello electrónico, cuyo certificado incorporado al recibo de salario verifica (junto con los requisitos del artículo 46.1 de la LPAC una vez se produzca su entrada en vigor) todas las garantías que debe ostentar para su validez, por lo que su autenticidad, integridad y conservación ${ }^{39}$ quedan perfectamente garantizadas.

\footnotetext{
${ }^{38}$ Debe tenerse en cuenta que cualquier comunicación electrónica se basa en el envío de paquetes de datos, todos los cuales están debidamente numerados e identificados por si alguno llega corrompido o simplemente erróneo, el propio sistema receptor (al efectuar la verificación de errores correspondiente) reclama al emisor dicho paquete, que es enviado nuevamente para su inclusión en la posición que corresponda dentro del conjunto de paquetes de datos que conforman el mensaje y, en su caso, documentos adjuntos al mismo.

${ }^{39}$ En relación con la conservación de los recibos de salario, debe tenerse en cuenta adicionalmente que el artículo 31 de la LAE prevé la existencia de archivos electrónicos de documentos los cuales, con-
} 
Como quiera que el documento administrativo electrónico será archivado también electrónicamente para su conservación, el artículo 18.2 de la LAE exige que se incluya el número de identificación fiscal y la denominación correspondiente, pudiendo contener la identidad de la persona titular del órgano administrativo, y debiendo cada Administración Pública adoptar las medidas adecuadas para facilitar la verificación de sus sellos electrónicos. Adicionalmente, con la entrada en vigor de la nueva legislación administrativa, habrán de tenerse en cuenta las previsiones de los artículos 46 de la LRJSP para los archivos electrónicos que se encuentren en fase activa, y 17 de la LPAC, para archivos (que deberían ser únicos) en fase de conservación.

En relación con la posibilidad de incluir el CSV en lugar del sello electrónico, debe recordarse que la seguridad jurídica que ofrece ha sido puesta en entredicho por autores como ALAMILLO ${ }^{40}$, sobre la base de que es un mecanismo que depende exclusivamente de la Administración que lo controla, por lo que si ésta quisiese borrarlo o modificarlo posteriormente a su notificación no habría forma de detectar dicha modificación ulterior, por lo que no se puede sino estar de acuerdo con tal extremo y recomendarse la utilización del sello electrónico en lugar del sistema de código CSV.

Por otra parte, adquiere especial relevancia el sistema de acceso al recibo de salario, ya que el mismo debe garantizar de forma adecuada y suficiente el principio de confidencialidad. En este sentido, debe apuntarse la posibilidad de utilizar un sistema de claves específicamente creado para ello, tal y como prevé el artículo 13.1 de la LAE: (esta previsión se contiene igualmente en el artículo 9.2.c de la LPAC, al regular los sistemas de identificación, y en el 10.2.c al regular los sistemas de firma electrónica, ya que en la nueva normativa se distinguen correctamente ambos, conforme con el Reglamento (UE) 910/2014).

\footnotetext{
forme con el apartado 3 de dicho precepto, deben ser seguros y observar la normativa vigente en materia de protección de datos, con el siguiente tenor literal: "los medios o soportes en que se almacenen documentos, deberán contar con medidas de seguridad que garanticen la integridad, autenticidad, confidencialidad, calidad, protección y conservación de los documentos almacenados. En particular, asegurarán la identificación de los usuarios y el control de accesos, así como el cumplimiento de las garantías previstas en la legislación de protección de datos". Tales requisitos se prevén así mismo en los artículos 17.3 de la LPAC y 46.3 de la LRJSP, para archivos que alberquen documentos en fase de conservación o activa, respectivamente, si bien en éstos se menciona, adicionalmente, la necesidad de observancia de los requisitos establecidos por el Esquema Nacional de Seguridad, aprobado por Real Decreto 3/2010.

${ }^{40}$ ALAMILLO DOMingO, I., en su comunicación presentada en el seno de la Comisión de Expertos para la reforma de la Administración Electrónica celebrada en el Centro de Estudios Políticos y Constitucionales (Madrid) el 4 de mayo de 2015 cuyo objeto fue el "Análisis de los anteproyectos de Ley del Procedimiento Administrativo Común (LPAC) y de la Ley de Régimen Furídico del Sector Público (LRFSP)".
} 
"Las Administraciones Públicas podrán determinar, teniendo en cuenta los datos e intereses afectados, y siempre de forma justificada, los supuestos y condiciones de utilización por los ciudadanos de otros sistemas de firma electrónica, tales como claves concertadas en un registro previo, aportación de información conocida por ambas partes u otros sistemas no criptográficos.

De conformidad con el apartado 2 de dicho precepto, para aquellos supuestos en los que se utilicen estos sistemas para confirmar información relativa a los conceptos salariales por una Administración Pública, ésta deberá garantizar la integridad y el no repudio por ambas partes de los documentos electrónicos concernidos. Esta posibilidad ha sido reconocida por la doctrina ${ }^{41}$, la cual ha puesto de manifiesto su adecuación y mejora respecto al envío por email, que se entiende menos garantista.

Como puede apreciarse, tales requisitos pueden ser observados por la firma electrónica, por lo que su utilización puede resultar incluso más garantista que el admitido sistema comentado anteriormente de claves de acceso al recibo de salario ubicado en los servidores de la intranet de la Administración, por lo que nada debe obstar para que las Administraciones Públicas lo implanten como forma normal de comunicación del recibo de salario.

\section{CONGLUSIONES}

Como se venía vaticinando ab initio del presente Trabajo, tanto los trabajadores como el moderno Derecho del Trabajo debe adaptarse a la novedosa realidad que proporcionan las nuevas tecnologías y beneficiarse de los aspectos positivos que supone la utilización de medios electrónicos, informáticos y telemáticos en las relaciones laborales, no sólo en el seno de empresas o entes privados, sino también en el ámbito de las Administraciones Públicas, con el fin de modernizar las relaciones y comunicaciones y el cumplimiento de las obligaciones derivadas de la relación laboral.

$\mathrm{Al}$ igual que en su día la implantación de las transferencias bancarias llegó a desplazar el cobro del salario en efectivo líquido a través de las "ventanillas" habilitadas a tal efecto y posterior ingreso bancario en la cuenta corriente de la que fuese

41 GÓMEZ-MILLÁN HERENCIA, M.J. (2012): 49, con expresa mención al sistema de acceso al recibo electrónico de salario a través de la intranet de la Administración. 
titular el trabajador por parte de éste ${ }^{42}$, en mi opinión con el recibo de salario ocurrirá igualmente, y desplazará de forma generalizada al recibo en formato papel, toda vez que los beneficios que suponen dicho formato resultan, a todas luces, evidentes e irrefutables, frente a escasas posibles oposiciones a su implantación, fundamentalmente basadas en la existencia de trabajadores desconocedores de asuntos relacionados con la informática o la ausencia de puestos de trabajo individuales informatizados para todos los trabajadores, que no obstante tienen solución (relativamente sencilla) y deberán ser superadas.

Es más, resulta llamativo que, en pleno siglo XXI, existan pronunciamientos jurisprudenciales que basen decisiones en la falta de equivalencia entre el formato electrónico y el papel, lo cual no hace sino poner de manifiesto la necesidad de una plena modernización y adaptación a la era digital no sólo de los ciudadanos y Administraciones Públicas, sino también de los poderes públicos encargados de la función jurisdiccional, puesto que ninguna duda cabe de la plena validez del principio de equivalencia funcional en esta materia, aspecto ampliamente superado.

Las reticencias a las nuevas tecnologías no sólo se manifiestan en el ámbito público, como se ha podido comprobar, sino que en el sector privado también surgen tanto a trabajadores como a sus representantes sindicales, a veces con fundamento suficiente y, en otras ocasiones, en ausencia del mismo.

Con la entrada en vigor de las Leyes 39 y 40/2015, de 1 de octubre, se produce además un verdadero cambio de paradigma que no hace sino confirmar lo aquí sostenido, al apostar ambas por el soporte electrónico como medio preferente en la actividad administrativa y, sobretodo, en la emisión de documentos administrativos; concretamente, el artículo 26.1 in fine de la LPAC establece que "las Administraciones Públicas emitirán los documentos administrativos por escrito, a través de medios electrónicos, a menos que su naturaleza exija otra forma más adecuada de expresión y constancia”, por lo que se confirma, a mi entender, la procedencia (incluso la obligación ex lege) de la emisión del recibo de salario en soporte electrónico, siempre que se cumplan los requisitos de validez establecidos en el apartado 2 del mismo precepto.

${ }^{42}$ Lo cual suponía en muchos casos, además, la necesidad para las Administraciones Públicas de conferir a los trabajadores permisos durante los últimos días del mes para poder acudir al cobro del salario en horas de trabajo, y la obligación de éstos de acudir físicamente a realizar tales trámites, a veces nada deseados. 
De todo lo expuesto no cabe sino colegir que la implantación del recibo electrónico de salario es una realidad que comienza a ser una exigencia de los principios de eficacia y eficiencia, entre otros, proclamados constitucionalmente, por lo que en las empresas públicas y privadas acabará desplazando al formato papel. En este sentido, los esfuerzos de los distintos agentes deben centrarse no en la lucha contra el nuevo sistema, sino en una verdadera colaboración para su implantación con todas las garantías que el actual estado de la técnica permiten actualmente, lo cual supondrá además, para determinados trabajadores, la superación de una verdadera barrera tecnológica que algunos han levantado frente a la utilización de las nuevas tecnologías.

\section{BIBLIOGRAFÍA}

ALAMILLO DOMINGO, I.: Comunicación presentada en el seno de la Comisión de Expertos para la reforma de la Administración Electrónica celebrada en el Centro de Estudios Políticos y Constitucionales (Madrid) el 4 de mayo de 2015 cuyo objeto fue el "Análisis de los anteproyectos de Ley del Procedimiento Administrativo Común (LPAC) y de la Ley de Régimen Furídico del Sector Público (LRFSP)".

CASTELLS, M. en su obra "La era de la información. La sociedad red". Alianza Editorial, Madrid (2005).

GAMERO CASADO, E. y FERNÁNDEZ RAMOS, S.: "Manual básico de Derecho Administrativo", Ed. Tecnos, $11^{\text {a }}$ ed. (2014).

GAMERO CASADO, E. y VALERO TORRIJOS, J. (Coord.), en "La Ley de Administración Electrónica. Comentario sistemático a la Ley 11/2007, de 22 de junio, de Acceso Electrónico de los Ciudadanos a los Servicios Públicos", Ed. Thomson Reuters Aranzadi, $3^{\text {a }}$ ed. (2010).

GAMERO CASADO, E. (coord.): "Simplificación del procedimiento administrativo y mejora de la regulación. Una metodología para la eficacia y el derecho a la buena administración", Ed. Tirant Lo Blanch (Valencia, 2014).

GÓMEZ-MILLÁN HERENCIA, M.J.: "El recibo de salario en formato electrónico. Comentario de la sentencia del Tribunal Supremo de 22 de junio de 2011". Boletín de Fun- 